\title{
Comportamiento de la mortalidad por dengue en Colombia entre 1985 y 2012
}

\author{
Pablo Chaparro-Narváez, Willian León-Quevedo, Carlos Andrés Castañeda-Orjuela \\ Observatorio Nacional de Salud, Instituto Nacional de Salud, Bogotá, D.C., Colombia
}

\begin{abstract}
Introducción. El dengue en Colombia es un importante problema de salud pública debido a las enormes pérdidas económicas y sociales que ha provocado, especialmente durante los picos epidémicos. Objetivo. Describir el comportamiento de la mortalidad por dengue en Colombia entre 1985 y 2012. Materiales y métodos. Se hizo un estudio descriptivo. La información se obtuvo de las bases de datos de mortalidad y de las proyecciones de población de 1985 a 2012 del Departamento Administrativo Nacional de Estadística (DANE). Se estimaron tasas de mortalidad, razón de tasas y letalidad.

Resultados. Se registraron 1.990 muertes por dengue durante el periodo señalado. Las tasas de mortalidad por dengue aumentaron entre 1985 y 1998. Las tasas más altas se presentaron en hombres menores de 5 años y de 65 o más años de edad. Entre 1995 y 2012 los municipios de categorías 1 a 4 también presentaron las tasas más altas. La letalidad durante el periodo varió entre 0,01 y 0,39 \%.

Conclusión. El dengue es una enfermedad evitable que debería desaparecer de las estadísticas de mortalidad como causa de defunción. La muerte es evitable en la medida en que se implementen y evalúen las actividades propuestas en la Estrategia de Gestión Integrada (EGI)-Dengue. Se recomienda impulsar el desarrollo de una cultura informática que contribuya a la toma de decisiones y a priorizar la utilización de los recursos asignados.
\end{abstract}

Palabras clave: dengue/mortalidad, registros de mortalidad, epidemiología, Colombia.

doi: http://dx.doi.org/10.7705/biomedica.v36i0.3009

Dengue mortality in Colombia, 1985-2012

Introduction: Dengue in Colombia is an important public health problem due to the huge economic and social costs it has caused, especially during the disease outbreaks.

Objective: To describe the behavior of dengue mortality in Colombia between 1985 and 2012.

Materials and methods: We conducted a descriptive study. Information was obtained from mortality and population projection databases provided by the Departamento Administrativo Nacional de Estadística (DANE) for the 1985-2012 period. Mortality rates, rate ratios, and case fatality rates were estimated.

Results: A total of 1,990 dengue deaths were registered during this period in Colombia. Dengue mortality rates presented an increasing trend with statistical significance between 1985 and 1998. Higher mortality rates were reported in men both younger than 5 years and older than 65 years. Between 1995 and 2012, category 1 to 4 municipalities reported the highest mortality rates. Case fatality rates varied during the period between $0.01 \%$ and $0.39 \%$.

Conclusion: Dengue is an avoidable disease that should disappear from mortality statistics as a cause of death. The event is avoidable if the proposed activities from the Estrategia de Gestión Integrada (EGI)-Dengue are implemented and evaluated. We recommend encouraging the development of an informational culture to contribute to decision making and prioritizing resource allocation.

Key words: dengue/mortality, mortality registries, epidemiology, Colombia.

doi: http://dx.doi.org/10.7705/biomedica.v36i0.3009

Correspondencia:

Pablo Chaparro-Narváez, Observatorio Nacional de Salud, Instituto Nacional de Salud, Avenida Calle $26 \mathrm{~N}^{\circ}$ 51-20, bloque B, oficina 208, Bogotá, D.C., Colombia

Teléfono: 220 7700, extensión 1389

pchaparro@ins.gov.co

Recibido: 30/07/15; aceptado: 10/02/16
La infección producida por el virus del dengue se considera como una de las enfermedades emergentes y reemergentes más importantes, ya que constituye una amenaza para un tercio de la población mundial (1). Entre los factores responsables de su emergencia y reemergencia,

\section{Contribución de los autores:}

Todos los autores participaron en el diseño del estudio, el análisis estadístico, la discusión de resultados y la redacción del manuscrito. 
se han identificado el crecimiento de la población, la urbanización no planificada, el incremento de los viajes aéreos, las migraciones y el deterioro de los programas de control $(2,3)$.

El dengue es una enfermedad infecciosa, endémica en muchos países tropicales y subtropicales, causada por un virus ARN del género Flavivirus (familia Flaviviridae), del cual se conocen cuatro serotipos, cada uno de ellos con capacidad de producir la enfermedad. El virus es transmitido a los humanos por la picadura del mosquito hembra infectado del género Aedes. Desde el punto de vista clínico, esta infección puede cursar asintomática $(4,5)$ o con una gran variedad de síntomas, con evolución y resultados impredecibles (6).

En el 2010, se estimaba que el dengue causó alrededor de 96 millones de infecciones aparentes a nivel mundial, de las cuales 66,8 millones ocurrieron en Asia, 15,7 millones en África, 13,3 millones en las Américas y 0,18 millones en Oceanía. Asimismo, se calculó que era el causante de 293,9 millones de infecciones no aparentes (7). En ese mismo año, fue el responsable de la pérdida de 825.000 (344.000 a 1'412.000) años de vida ajustados por discapacidad (AVAD), es decir, 12 (5 a 20) AVAD por cada 100.000 habitantes (8). Se ha establecido que 3.970 millones de personas en 128 países están en riesgo de infectarse con el virus del dengue (9).

El dengue ha sido una de las principales causas de hospitalización y muerte en los países asiáticos y de América Latina (10), y se ha establecido que anualmente es el responsable de cerca de 22.000 muertes en el mundo $(4,7,11)$, muchas de ellas asociadas con el dengue hemorrágico, o dengue con signos de alarma (12), y con el dengue grave (6). Se ha calculado que la tasa de mortalidad por dengue hemorrágico en la mayoría de los países es de alrededor de $5 \%$, y que los niños y adultos jóvenes son los más afectados (13), así como los ancianos y los pacientes con enfermedades concomitantes $(14,15)$ como la diabetes y la hipertensión (16). Entre los factores que se asocian con la muerte por dengue están los que facilitan la transmisión del virus, la gravedad de la enfermedad, las barreras de acceso a los servicios de salud, la residencia en municipios con una población menor de 100.000 habitantes y una edad de más de 65 años (17), así como la endemia, la precipitación anual, la densidad poblacional y la circulación del serotipo 2 , entre otros (18).
La mortalidad debida al dengue se considera evitable y su reducción depende principalmente de la detección precoz de los casos, el acceso oportuno a los servicios de salud y la calidad de la atención que se le proporcione a los afectados (19).

El dengue en Colombia es un importante problema de salud pública debido a las enormes pérdidas económicas $(20,21)$ y humanas que provoca, especialmente cuando se presentan epidemias. En el país, $74 \%$ de las cabeceras municipales están situadas por debajo de los 1.800 metros sobre el nivel del mar (msnm), y presentan condiciones climáticas, geográficas y epidemiológicas aptas para la transmisión de la enfermedad; de igual manera, se estima que cerca del $55 \%$ de la población colombiana se encuentra en riesgo de enfermar o morir por esta causa.

En el país se han hecho diversas investigaciones sobre aspectos relacionados con esta enfermedad. Sin embargo, a pesar de su reaparición, no se conoce el comportamiento de la mortalidad por dengue debido a que son escasos los estudios epidemiológicos sobre el tema. En este contexto, el objetivo de este estudio fue describir el comportamiento de las defunciones debidas a dengue, entre 1985 y 2012.

\section{Materiales y métodos}

Se hizo un estudio descriptivo para determinar el comportamiento de la mortalidad por dengue en Colombia entre 1985 y 2012. Los datos se obtuvieron de los registros de los certificados individuales de defunción consolidados en las bases de datos sobre mortalidad del Departamento Administrativo Nacional de Estadística (DANE).

Se analizaron las muertes codificadas como dengue de acuerdo con las normas de la Clasificación Internacional de Enfermedades (novena revisión para el periodo 1985-1996 y décima para el periodo 1997-2012) $(22,23)$. La codificación de las defunciones para el periodo de estudio cambió al código 0610 en la novena revisión, y a los códigos A90 y A91 en la décima revisión; en general, el diagnóstico de dengue se conservó.

La información sobre la población entre 1985 y 2012 se obtuvo de las estimaciones y proyecciones producidas por el DANE (24).

Las variables consideradas fueron: año de registro de la defunción, población total por año, número de muertes, sexo, edad, categoría del municipio de residencia habitual, municipio de residencia 
habitual y código de causa básica de defunción. La categoría del municipio empleada fue la señalada en la Ley 617 de 2000, la cual clasifica los municipios de acuerdo con el número de habitantes y los ingresos corrientes de libre destinación (cuadro 1) (25).

Se estimaron las tasas crudas de mortalidad por sexo y grupos de edad ( 0 a 4, 5 a 14, 15 a 44, 45 a 64 , y 65 y más años). Las tasas se presentaron como muertes por 100.000 personas al año.

Para comparar la evolución de la mortalidad en el tiempo, se calcularon las tasas anuales ajustadas por edad desde 1985 hasta 2012 utilizando el método directo y tomando como población de referencia la población nacional por grupos de edad y por quinquenios del censo de 2005. Se calculó la letalidad anual de los casos de dengue a partir de la información de los registros de morbilidad consolidados por el programa de control de vectores del Ministerio de Salud y de la Protección Social para los años 1985 a 1997 y de la información del Sistema de Vigilancia de Salud Pública (Sivigila) del Instituto Nacional de Salud para los años 1998 a 2012. Se calcularon razones de tasas con su respectivo intervalo de confianza de $95 \%$ para los periodos 1985-1994, 1995-2004 y 2005-2012, con el fin de comparar por grupos de edad y por categoría municipal.

La información sobre la circulación de los serotipos del virus de dengue se obtuvo de los consolidados del Laboratorio de Virología del Instituto Nacional de Salud.

Para el procesamiento de la información obtenida, se emplearon hojas de cálculo de Microsoft Excel ${ }^{\circledR}$. La información se analizó con el programa Stata ${ }^{\circledR}$, versión 12.

Esta investigación tuvo en cuenta los requisitos éticos establecidos en la Resolución 8430 de 1993 del Ministerio de Salud de Colombia, que en su artículo 11 establece que los estudios de este tipo son investigaciones "sin riesgo".

\section{Resultados}

Entre 1985 y 2012, se registraron 1.990 muertes por dengue en Colombia; 55,6 \% (1.106) de los fallecimientos se presentó en hombres. El promedio de edad fue de 29,7 años para hombres y de 30,4 años para mujeres. La relación entre hombres y mujeres fue de 1,25:1. La tasa cruda de mortalidad para el periodo de estudio fue de 0,18 muertes por 100.000 habitantes $(0,20$ para hombres y 0,16
Cuadro 1. Clasificación de los municipios colombianos

\begin{tabular}{ccc}
\hline $\begin{array}{c}\text { Categoría } \\
\text { municipal }\end{array}$ & $\begin{array}{c}\text { Tamaño de } \\
\text { la población } \\
\text { municipal }\end{array}$ & $\begin{array}{c}\text { Ingresos corrientes } \\
\text { de libre destinación } \\
\text { (SMLMV) }\end{array}$ \\
\hline 6 & $<10.000$ & Hasta 15.000 \\
5 & $10.001-20.000$ & $>15.000-25.000$ \\
4 & $20.001-30.000$ & $>25.000-30.000$ \\
3 & $30.001-50.000$ & $>30.000-50.000$ \\
2 & $50.001-100.000$ & $>50.000-100.000$ \\
1 & $100.001-500.000$ & $>100.000-400.000$ \\
Especial & $>500.000$ & $>400.000$ \\
\hline
\end{tabular}

SMLMV: salarios mínimos legales mensuales vigentes

Fuente: Ley 615 de 2000

para mujeres). Durante este periodo, las tasas de mortalidad presentaron grandes variaciones que permitieron establecer dos patrones, uno entre 1985 y 1996, en el que las tasas oscilaron entre 0,0 y 0,05 muertes por 100.000 habitantes, y otro entre 1997 y 2012, en el que las tasas variaron entre 0,15 y 0,60 casos por 100.000 habitantes (figura 1). En este último periodo, las tasas de mortalidad más altas correspondieron a los años 1998, 2000-2001, 2006 y 2010. Por sexo, las tasas de mortalidad fueron más altas en hombres (cuadro 2). Se estimó que las tasas de mortalidad de los hombres correspondieron a 1,31 $\left(\mathrm{IC}_{95 \%} 1,20-1,43\right)$ veces las tasas de la mortalidad en las mujeres. Para los grupos de edad de 75 a 79 años, y 80 y más años, las tasas de mortalidad de los hombres fueron de $1,55\left(\mathrm{IC}_{95 \%} 1,21-1,99\right)$ y $2,87\left(\mathrm{IC}_{95 \%} 2,34-\right.$ $3,51)$ veces las tasas de mortalidad de las mujeres, respectivamente.

La letalidad por dengue para el periodo de estudio fue de 0,20\%; en el 2000 se presentó la más alta con $0,39 \%$. Entre 1985 y 2012, circularon en el país los cuatro serotipos del virus del dengue (cuadro 2).

Las tasas de mortalidad específicas por grupo de edad evidenciaron un panorama complejo debido a las amplias variaciones durante el periodo (figura 2). Las tasas más altas se registraron en el grupo de 65 y más años de edad durante todo el periodo. A partir de 1998, las tasas de los menores de 5 años también fueron altas (cuadro 3).

La razón de tasas por grupos de edad de todo el periodo indicó que los grupos de 65 y más años y de 0 a 4 años presentaron un riesgo que superó en 9,82 y 6,86 veces el del grupo de 45 a 64 años, respectivamente. Al comparar las tasas de mortalidad por grupos de edad de los periodos 1985-1994 y 2005-2012, se presentó un 


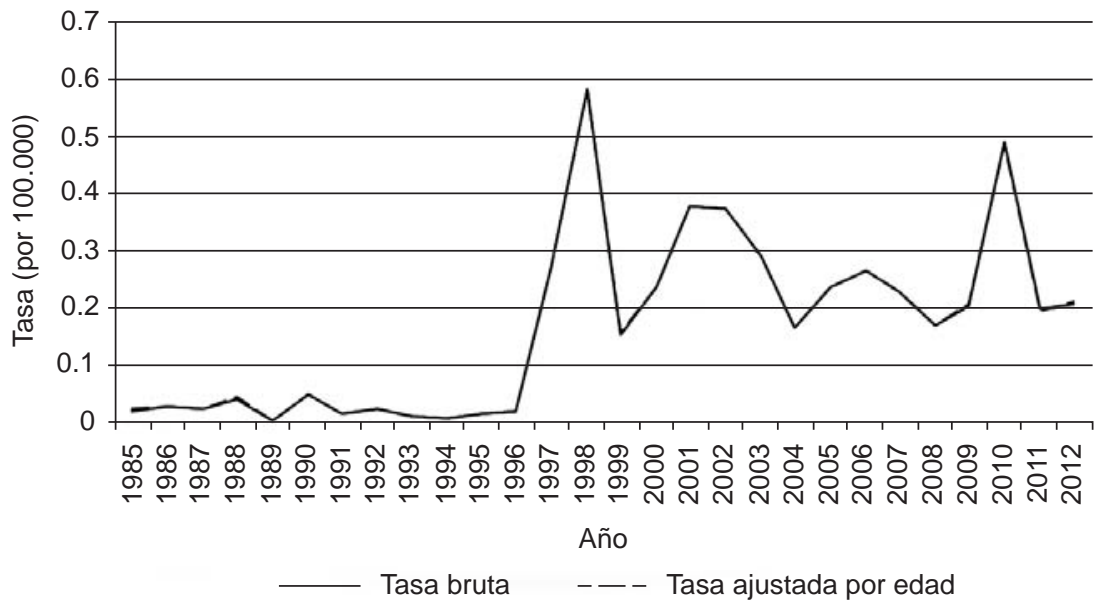

Figura 1. Tasas de mortalidad por dengue, Colombia, 1985-2012 Fuente: Estadísticas vitales, DANE

Cuadro 2. Tasas crudas de mortalidad por dengue según sexo, Colombia, 1985-2012

\begin{tabular}{|c|c|c|c|c|}
\hline \multirow{2}{*}{ Año } & \multicolumn{2}{|c|}{ Tasa de mortalidad } & \multirow{2}{*}{ Letalidad } & \multirow{2}{*}{$\begin{array}{l}\text { Serotipo circulante } \\
\text { reportado* }\end{array}$} \\
\hline & Hombre & Mujer & & \\
\hline 1985 & 0,02 & 0,02 & & DENV 1,2 \\
\hline 1986 & 0,03 & 0,03 & & DENV 1,2 \\
\hline 1987 & 0,02 & 0,02 & & DENV 1, 2 \\
\hline 1988 & 0,02 & 0,06 & & \\
\hline 1989 & 0,01 & 0,00 & & \\
\hline 1990 & 0,08 & 0,01 & 0,09 & DENV 1, 2, 4 \\
\hline 1991 & 0,00 & 0,03 & 0,03 & \\
\hline 1992 & 0,03 & 0,01 & 0,04 & DENV 2 \\
\hline 1993 & 0,01 & 0,02 & 0,02 & DENV 1, 2, 4 \\
\hline 1994 & 0,01 & 0,00 & 0,01 & \\
\hline 1995 & 0,02 & 0,01 & 0,01 & DENV 1, 2, 4 \\
\hline 1996 & 0,03 & 0,02 & 0,02 & \\
\hline 1997 & 0,29 & 0,26 & 0,44 & DENV 1, 2, 4 \\
\hline 1998 & 0,65 & 0,55 & 0,37 & DENV 1, 2 \\
\hline 1999 & 0,19 & 0,11 & 0,28 & \\
\hline 2000 & 0,30 & 0,19 & 0,39 & \\
\hline 2001 & 0,45 & 0,31 & 0,25 & DENV $1,2,4$ \\
\hline 2002 & 0,44 & 0,32 & 0,19 & DENV $1,3,4$ \\
\hline 2003 & 0,34 & 0,26 & 0,21 & DENV 1, 2, 3 \\
\hline 2004 & 0,19 & 0,14 & 0,29 & DENV 1, 2, 4 \\
\hline 2005 & 0,25 & 0,23 & 0,24 & DENV 1, 2, 3 \\
\hline 2006 & 0,32 & 0,22 & 0,30 & DENV $1,2,3,4$ \\
\hline 2007 & 0,26 & 0,20 & 0,23 & DENV 1, 2, 3, 4 \\
\hline 2008 & 0,14 & 0,20 & 0,20 & DENV $1,2,3,4$ \\
\hline 2009 & 0,25 & 0,16 & 0,17 & DENV $1,2,3,4$ \\
\hline 2010 & 0,54 & 0,45 & 0,14 & DENV 1, 2, 3, 4 \\
\hline 2011 & 0,22 & 0,18 & 0,30 & DENV $1,2,3,4$ \\
\hline 2012 & 0,25 & 0,17 & 0,18 & DENV $1,2,3,4$ \\
\hline
\end{tabular}

Fuentes: Estadísticas vitales, DANE; Programa de control de vectores, Ministerio de Salud y Protección Social; Sivigila, Instituto Nacional de Salud; *Laboratorio de Virología, Instituto Nacional de Salud

importante aumento en todos los grupos, el cual fue mayor en el de 5 a 14 años; al confrontar las tasas de mortalidad de los periodos 1995-2004 y
2005-2012, también se encontró un incremento en todos los grupos, aunque no tan acentuado como el encontrado para los periodos anteriores (cuadro 3). Las tasas de mortalidad por dengue más altas se hallaron en los municipios ubicados en la ribera del río Magdalena y, de forma aislada, en sectores de la Orinoquia, la Amazonia y la Costa Atlántica (figura 3).

La razón de tasas por categoría de municipio para el periodo 1985-1994 mostró que los municipios de categoría 3, 4 y 6 presentaron un riesgo de 8,10, 8,37 y 7,39 veces, respectivamente, en relación con los municipios de categoría especial. Para los periodos 1995-2004 y 2005-2012, la razón de tasas de los municipios de categorías 1 a 4 fue mayor que la de los municipios de categoría especial. Al comparar las tasas de mortalidad por categoría municipal entre los periodos 19851994 y 2005-2012, se registró un gran incremento en todas ellas, y el cambio más acentuado se dio en los municipios de categoría especial y en los de categorías 1 y 2 . Al comparar las tasas de mortalidad de 1995 a 2004 y de 2005 a 2012, se encontró una disminución en todas las categorías de municipios, especialmente en los de categoría 1,2 y 6 (cuadro 4).

\section{Discusión}

De acuerdo con los resultados de este estudio, las tasas de mortalidad por dengue en Colombia presentaron grandes variaciones y tendencia al incremento entre 1997 y 2012. En los hombres de los grupos de edad de menores de 5 años y de 65 y más años, las tasas de mortalidad fueron las más altas. Entre 1995 y 2012 los municipios de 


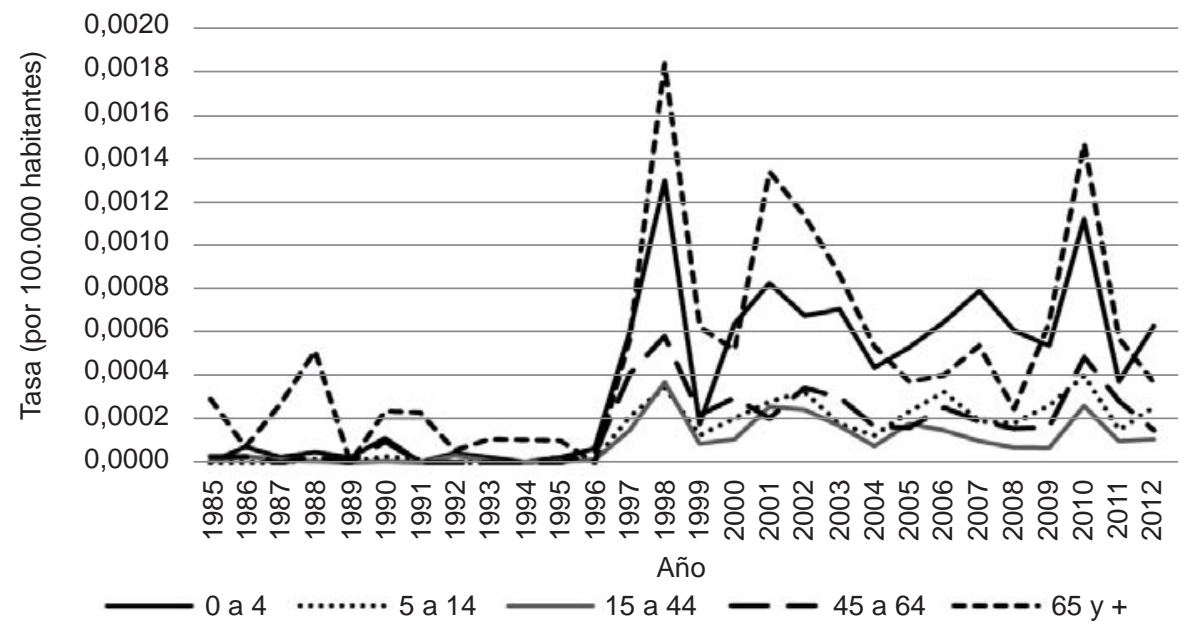

Figura 2. Tasas de mortalidad por dengue según grupo de edad, Colombia, 1985-2012 Fuente: Estadísticas vitales, DANE

Cuadro 3. Tasas de mortalidad por dengue según grupo de edad, Colombia, 1985-2012

\begin{tabular}{|c|c|c|c|c|c|c|c|c|}
\hline \multirow[t]{2}{*}{ Periodo } & \multirow{2}{*}{$\begin{array}{l}\text { Grupo } \\
\text { de edad }\end{array}$} & \multirow[t]{2}{*}{ Muertes } & \multirow[t]{2}{*}{ Población } & \multirow{2}{*}{$\begin{array}{l}\text { Tasa de } \\
\text { mortalidad }\end{array}$} & \multirow{2}{*}{$\begin{array}{c}\text { Razón de } \\
\text { tasas }\end{array}$} & \multicolumn{2}{|c|}{ Intervalo de confianza de $95 \%$} & \multirow[t]{2}{*}{ Variación } \\
\hline & & & & & & Inferior & Superior & \\
\hline & & & & & & & & $\begin{array}{c}1985-1994 \text { Vs } \\
2005-2012\end{array}$ \\
\hline \multirow[t]{5}{*}{ 1985-1994 } & 0 a 4 & 15 & $4 ’ 410.398$ & 0,34 & 7,68 & 3,6 & 16,4 & 1439,5 \\
\hline & 5 a 14 & 4 & $4^{\prime} 222.096$ & 0,09 & 2,14 & 0,64 & 7,10 & 2273,9 \\
\hline & 15 a 44 & 15 & 7’721.940 & 0,19 & 4,39 & 1,93 & 9,96 & 1144,3 \\
\hline & 45 a 64 & 7 & $15^{\prime} 804.837$ & 0,04 & 1 & & & 1470,1 \\
\hline & $65 y+$ & 30 & $1 ' 650.305$ & 1,82 & 41,04 & 25,17 & 66,92 & $\begin{array}{c}156,6 \\
1995-2004 \mathrm{Vs} . \\
2005-2012\end{array}$ \\
\hline \multirow[t]{5}{*}{$1995-2004$} & 0 a 4 & 246 & 4'533.372 & 5,43 & 6,97 & 5,84 & 8,31 & 108,8 \\
\hline & 5 a 14 & 161 & $5 ’ 648.191$ & 2,85 & 3,66 & 2,97 & 4,51 & 114,4 \\
\hline & 15 a 44 & 278 & 8'759.959 & 3,17 & 4,07 & 3,39 & 4,90 & 93,5 \\
\hline & 45 a 64 & 146 & $18^{\prime} 744.379$ & 0,78 & 1 & & & 111,9 \\
\hline & $65 y+$ & 176 & 2'288.754 & 7,69 & 9,87 & 8,26 & 11,79 & 110,8 \\
\hline \multirow[t]{5}{*}{ 2005-2012 } & 0 a 4 & 225 & 4'297.121 & 5,24 & 7,53 & 6,31 & 8,99 & \\
\hline & 5 a 14 & 176 & 7’825.628 & 2,25 & 3,23 & 2,63 & 3,98 & \\
\hline & 15 a 44 & 213 & 8'812.462 & 2,42 & 3,46 & 2,85 & 4,24 & \\
\hline & 45 a 64 & 145 & $20 ’ 851.315$ & 0,70 & 1 & & & \\
\hline & $65 y+$ & 137 & 2'936.907 & 4,66 & 6,71 & 5,48 & 8,21 & \\
\hline \multirow[t]{5}{*}{$1985-2012$} & 0 a 4 & 501 & 4'421.952 & 11,33 & 6,86 & 6,07 & 7,76 & \\
\hline & 5 a 14 & 352 & $5^{\prime} 760.996$ & 6,11 & 3,70 & 3,21 & 4,27 & \\
\hline & 15 a 44 & 516 & $8^{\prime} 404.239$ & 6,14 & 3,72 & 3,26 & 4,25 & \\
\hline & 45 a 64 & 302 & $18^{\prime} 296.524$ & 1,65 & 1 & & & \\
\hline & $65 y+$ & 364 & $2 ' 245.923$ & 16,21 & 9,82 & 8,68 & 11,11 & \\
\hline
\end{tabular}

Fuente: Estadísticas vitales, proyecciones y estimaciones de población, 1985-2020, DANE

categoría 1 a 4 también presentaron las tasas más altas. La letalidad durante el periodo varió entre 0,01 y $0,39 \%$.

Las variaciones de las tasas de mortalidad registradas probablemente sean el resultado de la confluencia de varios factores, entre los que se cuentan el comportamiento de esta enfermedad en el contexto latinoamericano, las distintas medidas adoptadas por los programas regional y nacional para la prevención y el control de la enfermedad, y la dinámica de la transmisión del virus.

Durante el siglo pasado, en la década de los años ochenta, la falta de sostenimiento y el abandono de los programas de control y erradicación de $A$. aegypti condujeron a la reinfestación de la mayoría de los países de la región de las Américas (26). En 


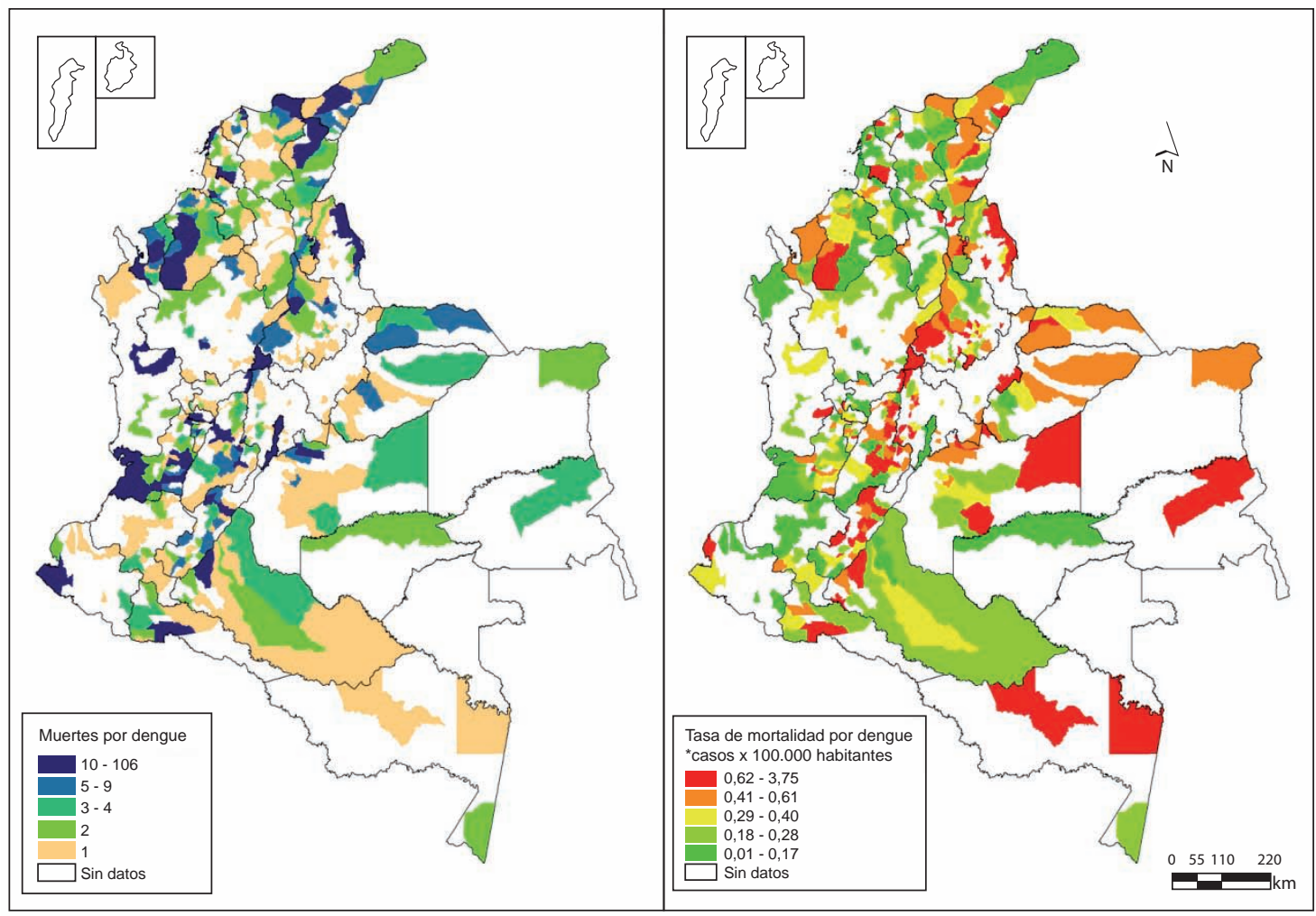

Figura 3. Mortalidad por dengue por municipios, Colombia, 1985-2012

Fuente: Estadísticas vitales, proyecciones y estimaciones de población, 1985-2020, DANE

Cuadro 4. Tasas de mortalidad por dengue según clasificación municipal, Colombia, 1985-2012

\begin{tabular}{|c|c|c|c|c|c|c|c|c|c|}
\hline \multirow[t]{2}{*}{ Periodo } & \multirow{2}{*}{$\begin{array}{l}\text { Categoría } \\
\text { municipal }\end{array}$} & \multirow{2}{*}{$\begin{array}{l}\text { Población } \\
\text { municipal }\end{array}$} & \multirow[t]{2}{*}{ Muertes } & \multirow[t]{2}{*}{ Población } & \multirow{2}{*}{$\begin{array}{c}\text { Tasa de } \\
\text { mortalidad }\end{array}$} & \multirow{2}{*}{$\begin{array}{c}\text { Razón de } \\
\text { tasas }\end{array}$} & \multicolumn{2}{|c|}{ Intervalo de confianza de $95 \%$} & \multirow[t]{2}{*}{ Variación } \\
\hline & & & & & & & Inferior & Superior & \\
\hline & & & & & & & & & $\begin{array}{c}1985-1994 \text { Vs. } \\
2005-2012\end{array}$ \\
\hline \multirow[t]{7}{*}{ 1985-1994 } & 6 & $<10.000$ & 7 & 2'035.146 & 0,34 & 7,39 & 2,78 & 19,63 & 460,5 \\
\hline & 5 & $10.001-20.000$ & 7 & 4'144.146 & 0,17 & 3,63 & 1,24 & 10,60 & 934,3 \\
\hline & 4 & $20.001-30.000$ & 12 & 3'079.870 & 0,39 & 8,37 & 3,51 & 19,99 & 486,3 \\
\hline & 3 & $30.001-50.000$ & 13 & 3’448.816 & 0,38 & 8,10 & 3,41 & 19,24 & 598,1 \\
\hline & 2 & $50.001-100.000$ & 7 & 3'178.709 & 0,22 & 4,73 & 1,67 & 13,39 & 1439,9 \\
\hline & 1 & $100.001-500.000$ & 19 & $7 ’ 176.260$ & 0,26 & 5,69 & 2,38 & 13,60 & 1409,1 \\
\hline & Especial & $>500.000$ & 5 & $10^{\prime} 746.628$ & 0,05 & 1 & & & $\begin{array}{c}3353,9 \\
1995-2004 \text { Vs. } \\
2005-2012\end{array}$ \\
\hline \multirow[t]{7}{*}{ 1995-2004 } & 6 & $<10.000$ & 46 & 2'385.860 & 1,93 & 1,20 & 0,87 & 1,65 & $-32,6$ \\
\hline & 5 & $10.001-20.000$ & 77 & 4'407.306 & 1,75 & 1,08 & 0,84 & 1,41 & $-3,5$ \\
\hline & 4 & $20.001-30.000$ & 75 & 3'283.345 & 2,28 & 1,42 & 1,09 & 1,85 & $-6,6$ \\
\hline & 3 & $30.001-50.000$ & 100 & 3’799.978 & 2,63 & 1,64 & 1,29 & 2,07 & 0,5 \\
\hline & 2 & $50.001-100.000$ & 130 & 3'833.692 & 3,39 & 2,11 & 1,71 & 2,61 & $-26,0$ \\
\hline & 1 & $100.001-500.000$ & 360 & 9'009.804 & 4,00 & 2,47 & 2,11 & 2,93 & $-31,4$ \\
\hline & Especial & $>500.000$ & 213 & $13 ’ 254.670$ & 1,61 & 1 & & & $-18,8$ \\
\hline \multirow[t]{7}{*}{ 2005-2012 } & 6 & $<10.000$ & 34 & 2’616.696 & 1,30 & 0,99 & 0,69 & 1,43 & \\
\hline & 5 & $10.001-20.000$ & 78 & $4^{\prime} 626.864$ & 1,69 & 1,29 & 0,99 & 1,68 & \\
\hline & 4 & $20.001-30.000$ & 76 & 3'562.125 & 2,13 & 1,64 & 1,26 & 2,13 & \\
\hline & 3 & $30.001-50.000$ & 109 & 4'120.929 & 2,65 & 2,03 & 1,61 & 2,55 & \\
\hline & 2 & $50.001-100.000$ & 110 & 4’381.248 & 2,51 & 1,93 & 1,53 & 2,42 & \\
\hline & 1 & $100.001-500.000$ & 287 & $10 ’ 463.497$ & 2,74 & 2,10 & 1,76 & 2,51 & \\
\hline & Especial & $>500.000$ & 195 & $14 ' 952.074$ & 1,30 & 1 & & & \\
\hline
\end{tabular}

Fuente: Estadísticas vitales, proyecciones y estimaciones de población, 1985-2020, DANE; Ley 615 de 2000 
la década de los noventa, a pesar de la exhortación al fortalecimiento de los programas nacionales y locales de prevención y lucha contra el dengue, se presentaron epidemias que afectaron a la mayoría de los países de la región, las cuales coincidieron con la circulación de todos los serotipos de dengue. En los albores del actual siglo, se adoptó en los países de la región de las Américas la Estrategia de Gestión Integrada para la Prevención y el Control del Dengue (EGI-Dengue) como medida para la prevención y el control de la enfermedad (27).

La dinámica de transmisión del virus, que obedece a la interacción entre el ambiente, el agente, la población de huéspedes y el vector, aunada a los distintos contextos sociales, culturales, económicos y políticos de las áreas donde la enfermedad se presenta, fue la responsable de las epidemias de los años 1987, 1990, 1993, 1995, 1998, 2002 y 2010, de las cuales la pandemia de 1998 produjo el más alto número de muertes que se haya registrado en el país.

En los tres periodos analizados, los municipios con diferente nivel de desarrollo presentaron, en general, tasas de mortalidad más altas que los municipios de categoría especial; sin embargo, la razón de las tasas de mortalidad fue estadísticamente significativa para los municipios de categorías 1 a 4 en los periodos 1995-2004 y 2005-2012. Es posible que en los municipios de categorías 1 a 3 las actividades de prevención y control de vectores, a cargo del nivel municipal, no fueran suficientes, pero en los de categoría 4 , en los cuales esta responsabilidad es de competencia del nivel departamental, tampoco resultó efectiva (28).

Independientemente de la categoría del municipio, la gran mayoría experimentó un crecimiento de la población, y se vio afectada por la migración del campo a la ciudad y por la urbanización no planificada, a lo cual hay que añadir la insuficiente infraestructura urbana básica, las viviendas con condiciones deficientes, y la falta de un adecuado suministro de servicios como agua, alcantarillado y recolección de basuras $(29,30)$. Por otra parte, la proliferación masiva de productos no reciclables, como plásticos no retornables, envases y neumáticos, condujo a un deterioro del medio ambiente que favoreció el aumento de los criaderos de mosquitos (31).

Si a estos factores se suman el incremento de la migración de individuos a áreas endémicas para el dengue, el deterioro de las medidas de prevención y control del vector, y los limitados recursos económicos y humanos destinados a las actividades de salud pública, se comprende la razón por la cual el dengue se estableció como una enfermedad persistente en la población colombiana (3).

Se evidenciaron mayores tasas de mortalidad por dengue en los municipios con menor densidad poblacional y menores ingresos económicos (municipios de categoría 6) que en los municipios con mayor densidad poblacional y mayores ingresos económicos (municipios de categoría especial). La Ley 715 del 2001 estableció como competencias de los departamentos del país la implementación de las acciones de control de vectores en los municipios de categorías 4, 5 y 6 de su jurisdicción, y como competencias de los distritos y municipios de categoría especial, y de categorías 1, 2 y 3, la formulación y la ejecución de las acciones de promoción, prevención, vigilancia y control de vectores (28). Con estas disposiciones es posible que se hayan debilitado las actividades de control habitual en los municipios de categoría 6 debido a que los departamentos redujeron sus actividades en este campo. Es probable que estos últimos municipios no contaran con los recursos humanos, físicos y financieros para asumir las responsabilidades, lo cual también se ha evidenciado en los procesos de reforma y descentralización implementados en América Latina (32).

Se considera que la calidad de la atención médica, el acceso oportuno a los servicios de salud, la promoción y la prevención son elementos básicos para reducir la mortalidad por dengue (19). En Colombia, la reforma y la descentralización separaron estas actividades, de manera que las de promoción y prevención quedaron a cargo de las secretarías locales de salud, y las de diagnóstico y tratamiento, en manos de las aseguradoras y las instituciones prestadoras de servicios de salud.

Otros factores que también han podido contribuir al aumento de la mortalidad son la ocupación, la pobreza, el analfabetismo, los conocimientos y las prácticas de la comunidad en relación con la enfermedad, la desnutrición, la falta de acceso al diagnóstico y la disponibilidad y la oportunidad de tratamiento, y la capacidad y calidad de los mismos servicios de salud (33). Entre las prácticas inadecuadas relacionadas con las medidas preventivas y de control de la enfermedad, figura el almacenamiento de agua, una práctica común 
que, independientemente de la condición socioeconómica de la población, se da como respuesta a las interrupciones del suministro y a su precio elevado en algunos lugares (34).

Las tasas de mortalidad por grupo de edad fueron más altas entre los menores de 5 años y mayores de 64 años. Patrones similares se han reportado en México (35) y Tailandia, aunque en periodos diferentes $(36,37)$. En los grupos de más edad, el riesgo de morir aumenta y probablemente está asociado con el incremento del número de enfermedades concomitantes y con una mayor propensión a contraer infecciones en general $(38,39)$; también se ha aducido la transición demográfica (40).

Los hallazgos de este estudio tienen implicaciones para las estrategias de prevención y control del dengue. El modelo de gestión EGI-Dengue tiene como objetivo fortalecer los programas nacionales para reducir la morbilidad, la mortalidad, y la carga social y económica que produce el dengue. Sin embargo, se han señalado problemas y debilidades relacionados con la gerencia del programa, con la vigilancia epidemiológica y entomológica y el control del vector, con la atención de los pacientes y el servicio de laboratorio, y con la comunicación social, aspectos que deben ser revisados y corregidos.

Otros factores que pueden explicar la muerte debida a dengue son los que tienen que ver con las prácticas clínicas en la atención de los pacientes, principalmente los relacionados con la atención en la primera consulta, en la que el diagnóstico puede pasar desapercibido, así como con el uso de pruebas inapropiadas y la interpretación de sus resultados (6).

Los resultados que se presentan deben interpretarse con precaución debido a que provienen de una fuente de información secundaria, lo cual implica limitaciones en la calidad de los datos (cobertura y contenido), así como en su completitud y validación. En relación con la cobertura, el subregistro puede ser consecuencia de las dificultades para determinar la causa exacta de la muerte. La causa básica de defunción podría quedar enmascarada por una inadecuada elaboración de las causas en el certificado de defunción, debido a que se pueden registrar los eventos derivados de las complicaciones que produce el dengue o las enfermedades que se consideran en su diagnóstico diferencial (41). Igualmente, es posible que al establecer la causa del fallecimiento no se tenga en cuenta la definición de muerte por dengue establecida en el protocolo de vigilancia en salud pública desde la década pasada (42). A pesar de que en los últimos años la vigilancia de esta enfermedad ha incorporado el análisis de los casos de muerte, aún falta comparar y validar la información recopilada en los certificados de defunción correspondientes a defunciones por dengue.

Por otra parte, puede haber dificultades para el cálculo preciso de las tasas de mortalidad relacionadas con problemas que afectan el numerador o el denominador. El numerador puede verse influido por el subregistro en las defunciones, en tanto que el denominador puede verse afectado por la población considerada para la estimación, ya sea la población total o la población en riesgo. En este estudio se utilizó la primera, con lo cual pudo haberse diluido la magnitud de la enfermedad. La población en riesgo es difícil de establecer si se tiene en cuenta la altitud de los municipios considerados endémicos, así como el vector.

A pesar de las limitaciones, en este estudio se analizó el comportamiento de la tendencia de la mortalidad por dengue y puede ser la base para llevar a cabo investigaciones que utilicen otras metodologías y que permitan ampliar el conocimiento sobre la mortalidad por dengue en la población colombiana en riesgo.

En conclusión, el dengue es una enfermedad evitable que debería desaparecer de las estadísticas de mortalidad como causa de defunción. La muerte puede evitarse en la medida en que se implementen y evalúen las actividades propuestas en la EGI-Dengue, como la vigilancia epidemiológica; la implementación de prácticas eficaces para el diagnóstico del dengue en las redes de laboratorios; la vigilancia y el control de los vectores; el manejo clínico de los pacientes, y la comunicación y la movilización social (27).

Asimismo, se recomienda impulsar el desarrollo de una cultura informática entre los responsables de la vigilancia en salud pública, la cual deberá incorporar las estadísticas vitales y la información del programa de prevención y control de las enfermedades transmitidas por vectores, con el fin de conocer en tiempo real los casos de mortalidad con base en el certificado de defunción y de que se mejore la calidad de los datos que se consignan. Aunque aún hay retrasos y limitaciones en la consolidación nacional de la información proveniente de los certificados de defunción, estos son la principal 
fuente de información sobre la mortalidad y contribuyen a la adopción de decisiones y a priorizar la utilización de los recursos asignados.

\section{Conflicto de intereses}

Los autores declaran que no existe conflicto de intereses.

\section{Financiación}

Este trabajo se hizo con los recursos de funcionamiento del Observatorio Nacional de Salud del Instituto Nacional de Salud.

\section{Referencias}

1. Lemos G. Dengue, un problema social reemergente en América Latina. Estrategia para su erradicación. Biotecnología Aplicada. 2006;23:130-6.

2. Guzmán MG, Kouri G. Dengue and dengue hemorrhagic fever in the Americas: Lessons and challenges. J Clin Virol. 2003;27:1-13.

3. Gubler DJ. Epidemic dengue/dengue hemorrhagic fever as a public health, social and economic problem in the 21st century. Trends Microbiol. 2002;10:100-3. http://dx.doi. org/10.1016/S0966-842X(01)02288-0

4. Simmons CP, Farrar JJ, Nguyen vV, Wills B. Dengue. N Engl J Med 2012;366:1423-32 http://dx.doi.org/10.1056/ NEJMra1110265

5. Endy TP, Anderson KB, Nisalak A, Yoon IK, Green $\mathrm{S}$, Rothman AL, et al. Determinants of inapparent and symptomatic dengue infection in a prospective study of primary school children in Kamphaeng Phet, Thailand. PLoS Negl Trop Dis. 2011;5:e975. http://dx.doi.org/10.1371/ journal.pntd.0000975

6. World Health Organization (WHO). Dengue: Guidelines for diagnosis, treatment, prevention and control: New edition. WHO: Geneva; 2009.

7. Bhatt S, Gething P, Brady O, Messina J, Farlow A, Moyes $\mathbf{C}$, et al. The global distribution and burden of dengue. Nature 2013;496:504-7 http://dx.doi.org/:10.1038/ nature 12060

8. Murray C, Vos T, Lozano R, Naghavi M, Flaxman A, Michaud C, et al. Disability-adjusted life years (DALYs) for 291 diseases and injuries in 21 regions, 1990-2010: A systematic analysis for the Global Burden of Disease Study 2010. Lancet. 2012;380:2197-223 http://dx.doi. org/10.1016/S0140-6736(12)61689-4

9. Brady OJ, Gething PW, Bhatt S, Messina JP, Brownstein JS, Hoen AG, et al. Refining the global spatial limits of dengue virus transmission by evidence-based consensus. PLoS Negl Trop Dis. 2012;6:e1760. http://dx.doi.org/10. 1371/journal.pntd.0001760

10. World Health Organization. Dengue and severe dengue (Fact sheet N117). Fecha de consulta: 15 de abril de 2015. Disponible en: http://www.who.int/mediacentre/factsheets/ fs $117 /$ en/

11. Centers for Disease Control and Prevention. Dengue Homepage. Fecha de consulta: 1ํ de mayo de 2015. Disponible en: http://www.cdc.gov/Dengue/
12. World Health Organization. Dengue hemorrhagic fever: Diagnosis, treatment, prevention and control. Second edition. Geneva: World Health Organization; 1997. p. 84.

13. Ganguly A, Malabadi RB, Bhatnagar PK, Tang X, Das $\mathbf{D}$, Loebenberg $\mathbf{R}$, et al. Production and characterization of monospecific and bispecific antibodies against dengue virus NS1 protein. J Virol Methods. 2015;220:5-12. http:// dx.doi.org/10.1016/j.jviromet.2015.04.004

14. World Health Organization. Handbook for clinical management of dengue. Geneva: World Health Organization; 2012. p. 111.

15. Ministério da Saúde. Dengue: diagnóstico e manejo clínico: adulto e criança. 4th edition. Brasilia: Ministério da Saúde; 2013. p. 80.

16. Karunakaran A, Ilyas WM, Sheen S, Jose N, Nujum Z. Risk factors of mortality among dengue patients admitted to a tertiary care setting in Kerala, India. J Infect Public Health. 2014;7:114-20. http://dx.doi.org/10.1016/j.jiph.2013.09.006

17. Campos KB, Amâncio FF, de Araújo VE, Carneiro M. Factors associated with death from dengue in the state of Minas Gerais, Brazil: Historical cohort study. Trop Med Int Health. 2015;20:211-8. http://dx.doi.org/10.1111/tmi.12425

18. Díaz-Quijano FA, Waldman EA. Factors associated with dengue mortality in Latin America and the Caribbean, 19952009: An ecological study. Am J Trop Med Hyg. 2012;86: 328-34. http://dx.doi.org/10.4269/ajtmh.2012.11-0074.

19. Martínez E. La prevención de la mortalidad por dengue: un espacio y un reto para la atención primaria de salud. Rev Panam Salud Pública. 2006;20:60-74. http://dx.doi. org/10.1590/S1020-49892006000700013

20. Shepard D, Coudeville L, Halasa Y, Zambrano B, Dayan G. Economic impact of dengue illness in the Americas. Am J Trop Med Hyg. 2011;84:200-7. http://dx.doi.org/10.4269/ ajtmh.2011.10-0503

21. Castañeda-Orjuela C, Díaz H, Álvis-Guzmán N, Olarte A, Rodríguez H, Camargo G, et al. Burden of disease and economic impact of dengue and severe dengue in Colombia, 2011. Value Health Reg Issues. 2012;1:123-8. http://dx.doi. org/10.1016/j.vhri.2012.09.014

22. Organización Panamericana de la Salud. Manual de la clasificación estadística internacional de enfermedades, traumatismos y causas de defunción. Novena revisión. Washington, D.C.: OPS; 1978.

23. Organización Panamericana de la Salud. Clasificación estadística internacional de enfermedades y problemas relacionados con la salud. Décima revisión. Washington, D.C.: OPS; 1995.

24. Departamento Administrativo Nacional de Estadística. Estimaciones 1985-2005 y proyecciones 2005-2020 nacional y departamental desagregadas por sexo, área y grupos quinquenales de edad. Fecha de consulta: 10 de mayo de 2015. Disponible en: http://www.dane.gov.co/index.php/ poblacion-y-demografia/proyecciones-de-poblacion

25. Ministerio de Hacienda y Crédito Público. Ley 617 de 2000. Fecha de consulta: 30 de mayo de 2015. Disponible en: http://www.minhacienda.gov.co/portal/page/ portal/HomeMinhacienda/asistenciaentidadesterritoriales/ Publicaciones/Libros/Ley\%20617\%20de\%20200\%20 version\%202008.pdf 
26. Organización Panamericana de la Salud. Plan continental de ampliación e intensificación del combate al Aedes aegypti. Rev Panam Salud Pública. 1998;3:124-30. http:// dx.doi.org/10.1590/S1020-49891998000200014

27. Organización Panamericana de la Salud, Organización Mundial de la Salud. Últimos adelantos técnicos en la prevención y el control del dengue en la Región de las Américas. Informe de reunión. Washington. 2014. Fecha de consulta: 24 de mayo de 2015. Disponible en: http:// www.slamviweb.org/es/home/dengue/2014-cha-adelantosprevencion-americas-dengue.pdf

28. Ministerio de Hacienda y Crédito Público. Ley 715 de 2001. Por la cual se dictan normas orgánicas en materia de recursos y competencias de conformidad con los artículos 151,288, 356 y 357 de la Constitución Política y se dictan otras disposiciones para organizar la prestación de los servicios de educación y salud, entre otros. Bogotá D.C.: Congreso de la República; 2001. Fecha de consulta: 30 de mayo de 2015. Disponible en: http://www. minhacienda.gov.co/portal/page/portal/HomeMinhacienda/ presupuestogeneraldelanacion/Normativapresupuesto/ Leyes/Ley\%20715\%20de\%20diciembre\%2021\%20 de $\% 202001$

29. Barrera R, Ávila J, González S. Unreliable water supply of potable water and elevated Aedes aegypti larval indices: A causal relationship. J Am Mosq Control Assoc. 1993;9: 189-95.

30. Toan DT, Hoat LN, Hu W, Wright P, Martens P. Risk factors associated with an outbreak of dengue fever/dengue haemorrhagic fever in Hanoi, Vietnam. Epidemiol Infect. 2015; 143:1594-8. http://dx.doi.org/10.1017/S0950268814002647

31. Gómez-Dantés H, Willoquet J. Dengue in the Americas: Challenges for prevention and control. Cad Saúde Pública. 2009;25:S19-31. http://dx.doi.org/10.1590/S0102311X2009001300003

32. Barón G. Cuentas de salud de Colombia 1993-2003: el gasto nacional en salud y su financiamiento. Bogotá: Ladiprint Editorial Ltda.; 2007. p. 155.

33. Starfield B. Atenção primária: equilíbrio entre necessidades de saúde, serviçios e tecnologia. Brasília: UNESCO, Ministerio de Saúde; 2002.
34. García-Betancourt T, Higuera-Mendieta D, GonzálezUribe C, Cortés S, Quintero J. Understanding water storage practices of urban residents of an endemic dengue area in Colombia: Perceptions, rationale and socio-demographic characteristics. PLoS One. 2015;10:e0129054. http://dx.doi. org/10.1371/journal.pone.0129054

35. Gaxiola-Robles R, Celis A, Serrano-Pinto V, OrozcoValerio M, Zenteno-Savín T. Mortality trend by dengue in México 1980 to 2009. Revista de Investigación Clínica. 2012;64:444-51.

36. Kongsomboon K, Singhasivanon $P$, Kaewkungwal J, Nimmannitya S, Mammen MP Jr, Nisalak A, et al. Temporal trends of dengue fever/dengue hemorrhagic fever in Bangkok, Thailand, from 1981 to 2000: An age-periodcohort analysis. Southeast Asian J Trop Med Public Health. 2004;35:913-7.

37. Halstead S. More dengue, more questions. Emerg Infect Dis. 2005;11:740-1. http://dx.doi.org/10.3201/eid1105.050346 PMCID: PMC3320355

38. García-Riveira EJ, Rigau-Pérez JG. Dengue severity in elderly in Puerto Rico. Rev Panam Salud Pública. 2003;13: 362-8. http://dx.doi.org/10.1590/S1020-49892003000500004

39. Lee IK, Liu JW, Yang KD. Clinical and laboratory characteristics and risk factors for fatality in elderly patients with dengue hemorrhagic fever. Am J Trop Med Hyg. 2008;79:149-53.

40. Guha-Sapir D, Schimmer B. Dengue fever: New paradigms for a changing epidemiology. Emerg Themes Epidemiol. 2005;2:1. http://dx.doi.org/10.1186/1742-7622-2-1

41. Arbo A, Basualdo W, Bogado N, Iramain R, Lovera D, Pavlicih V. Manejo de casos de dengue durante epidemias. Pediatr (Asunción). 2011;38:57-62.

42. Instituto Nacional de Salud. Dengue. Protocolo de vigilancia en salud pública. 2014. Fecha de consulta: 30 de mayo de 2015. Disponible en: http://www.ins.gov.co/lineasde-accion/Subdireccion-Vigilancia/sivigila/Protocolos $\% 20$ SIVIGILA/PRO\%20Dengue.pdf 